Bayero Journal of Pure and Applied Sciences, 12(1): 5 -12

ISSN $2006-6996$

\title{
STUDIES ON SOME IMMUNOLOGICAL PROFILE OF PATIENTS INFECTED WITH TUBERCULOSIS ATTENDING INFECTIOUS DISEASES HOSPITAL, KANO
}

\author{
Aminu, A. I. ${ }^{1 *}$ and Habib, N. L. ${ }^{2}$ \\ ${ }^{1}$ Department of Microbiology, Bayero University, Kano, Nigeria \\ ${ }^{2}$ Infectious Diseases Hospital, Kano, Nigeria \\ Correspondence author:*aishatuaminuibrahim@gmail.com, 08054503326.
}

ABSTRACT

The magnitude of the immune response to tuberculosis (TB) infection also involves cellmediated immunity comprising numerous types of T-cell subsets and this reflects the magnitude of the bacterial load and those with the highest load are at a greater risk of developing TB disease. The study assesses the immunological profile (CD4t, CD8t, $C D 3+, C D 45+$ ) of TB infected individuals with drug sensitive TB (DS-TB) and compares it with that of subjects with Latent Tuberculosis Infection (LTBI) and those with Rifampicin Resistant TB (RR-TB). The study was a cross-sectional study conducted at Infectious Diseases Hospital (IDH), Kano. Two hundred and five (205) subjects participated in the study. Collected sputum samples were subjected to Ziehl-Neelsen (ZN) staining and cultured on Lowenstein Jensen medium for the identification of Mycobacteria tuberculosis. Molecular detection of M. tuberculosis and RR-TB was performed using Xpert ${ }^{B}$ MTB/RIF. Blood samples were also collected and the T-cell counts were determined using flow cytometry. The result of the study indicated that 131 of the studied subjects were males and 74 were females and that majority of them were in the group 14-24years (41.0\%), 25-34 years (35.6\%) and 35-44 years (13.7\%) respectively. Among the DS-TB and RR-TB groups majority were males $(70.8 \%$ and $66.7 \%$ respectively). It was shown that $86.2 \%$ and $88.9 \%$ of the samples from the DS-TB and $R R-T B$ were AFB positive. However, all the 65 samples of DS-TB and 54 samples of the $R R-T B$ were identified as MTB by culture. All the DS-TB samples were detected as susceptible TB by Xpert@ MTB/RIF, and all the samples of the RR-TB were confirmed as rifampicin resistant by the Xpert $\mathbb{B}$ MTB/RIF. The study also revealed that the $T$-cell $(C D 4+, C D 8+, C D 3+$ and $C D 45+)$ counts of the studied subjects varied significantly among the different groups of the study $(p<0.05)$ and that compared to the control and the $L T B I$ groups the DS-TB and the RR-TB groups recorded the lowest counts in the order; Control>LTBI>DS-TB>RR-TB.

Key words: Tuberculosis, T-cells counts, Drug susceptible TB, Latent TB, RR-TB.

\section{INTRODUCTION}

Tuberculosis (TB) remains one of the greatest health problems affecting people especially those living in developing countries (World Health Organisation [WHO], 2018). The development of drug resistance (DR) TB including multidrug resistant TB (MDR-TB), extensively DR-TB (XDR-TB) and totally DR-TB (XXDR-TB) further worsen the situation. Currently, the WHO reveals that an estimated 10 million people are infected with TB and 1.3 million deaths occurs worldwide in 2017 (WHO, 2018). Globally, an estimated $600,000 M D R /$ Rifampicin resistant-TB or RR-TB cases were reported with $3.5 \%$ of new TB cases and $18 \%$ of previously treated cases identified as MDR/RR-TB. Among cases of MDR-TB in $2017,8.5 \%$ were estimated to have extensively drug-resistant TB (XDR-TB) (WHO, 2018 \& 2017). Nigeria records $4.3 \%$ and $25 \%$ MDRTB/RR-TB among new and old TB cases (WHO, 2018).

Studies revealed that TB infection does not necessarily lead to disease, only 3 to $10 \%$ of immune competent individuals that are infected will TB develop the disease during their life time, while more than $90 \%$ of infected subjects contain the infection in form of sub-clinical stage known as Latent Tuberculosis Infection (LTBI) in which the pathogen remain in quiescent state (McNerney et al., 2012; Zumla et al., 2010). 


\section{Special Conference Edition, November, 2019}

Besides the initial physical defense that prevents TB infection in most persons exposed to tuberculosis, it was reported that protection against $M$. tuberculosis infection is based on the cell-mediated immunity (CMI) involving $\mathrm{CD}^{+}$ and $\mathrm{CD}^{+}{ }^{+}$-cell subsets, though macrophages and dendrintic cells also play important role in controlling TB infection (Schoenborn and Wilson, 2007; Deveci, 2006). CD4 T-cells recognise antigenic peptides derived from the phagosomal compartment in the context of MHC-class II molecule and it was further illustrated that Th 1 and Th 17 CD4 $\mathrm{T}$ cells which are the most abundant immune effector cells present in alveolar spaces have been involved in protection against TB at early stages for their capacity to recruit monocytes to the site of granuloma (Perrau, 2013; Kaufmann, 2013). Similarly, on specific antigen recognition CD8 T-cells differentiate into effector cells which produce cytolytic molecules and cytokines that kill both host cells and intracellular M. tuberculosis (Kaufmann, 2013). CD8 T-Lymphocytes recognise antigenic peptides which are generally loaded in the cytolytic compartment in the context of MHC class-I molecules (Dorhoi, et al., 2012). Doherty and Andersen (2005) reiterated that the outcome of infection relies on a balance between antigen-specific inflammatory and regulatory T cells in both the MHC I- and MHC II-restricted T-cell compartments.

In another study, Kaufmann (2013) illustrated that immunological activity against $M$. tuberculosis also involves several conventional and unconventional T-cell subsets that are characterised by distinct effector functions and surface phenotype markers. Among them, detailed analysis of paraformaldehyde-fixedhuman tuberculosis tissues revealed that apoptotic CD3+ and CD45 RO+ cells are present in productive tuberculosis granulomas, particularly those harbouring a necrotic center (Fayyazi et al., 2000). CD3 is a surface marker molecule that characterise human cells involved in immune response and present on all $\mathrm{T}$ cells (Davey, 1993). CD45 is a tyrosine phosphate common to all hematopoietic cells and identifies leucocytes (Willey, 2008).

Earlier studies by Doherty and Andersen (2005) suggested that the magnitude of the immune response to TB infection reflects the magnitude of the bacterial load and that those with highest load are at a greater risk of developing TB disease. In an attempt to establish whether the immunological markers could be used to differentiate TB infection stages (latent or active TB) and type of TB diseases (susceptible TB or $R R-T B$ ), the study assesses the immunological profile $(\mathrm{CD} 4+, \mathrm{CD} 8+, \mathrm{CD} 3+, \mathrm{CD} 45+)$ of $\mathrm{TB}$ infected individuals with drug sensitive TB (DSTB) and compares it with that of subjects with Latent Tuberculosis Infection (LTBI) and those with Rifampicin resistance. Such a study would not only allow early identification of patients that would likely develop the disease but also allow patients to be stratified into different groups according to disease severity as such allowing drug regimens to be administered appropriately which enhances adherence and treatment outcome.

\section{MATERIALS AND METHODS}

The study area was Kano state in Northern Nigeria. It is located within Savanna, latitude 120 to $12015^{\prime} \mathrm{N}$ and longitude 80301 to $451 \mathrm{E}$. It has an elevation of about 525 meters above the sea level with a population of 12.4 million (NIPOST, 2008).

The study was a cross-sectional study conducted at the Infectious Diseases Hospital (IDH), Kano. Ethical approval for the study was obtained from the Research Ethics Committee of Kano State Hospitals Management Board. An informed consent was also sought from the participants of the study prior to sample collection. Subjects with documented history of HIV infection and other immunocomprised conditions and those who did not provide consent were excluded from the study.

The participants who consented to participate in the study are grouped into four groups; the first group consists of 46 healthy volunteers who have non-detectable delayed-type hypersensitivity (DTH) intradermal reaction to purified protein derivatives (PPD) and serve as the control. The second group consists of 40 health care workers who have a positive DTH response to PPD defining a latent TB Infection (LTBI) group. The third group consists of 65 patients who have been recently diagnosed with active pulmonary drug sensitive TB (DS-TB), the fourth group consist of 54 patients with rifampicin resistant-TB (RR-TB). All the subjects were sero-negative to human immunodeficiency virus (HIV) infection and non-diabetic.

\section{Determination of TB status}

Sputum samples were collected and processed according to standard mycobacteiological procedures described by National Tuberculosis and Leprosy Control Programme (NTBLC) SOP Manual (2011) and National Committee for Clinical Laboratory Standards (NCCLS) (2011). Accordingly, the collected sputum samples were liquefied with $\mathrm{N}$-acetyl L-cystein (NALC), decontaminated with $\mathrm{NaOH}$ solution and neutralized with Phosphate Buffered Saline (PBS) solution and then centrifuged. 


\section{Special Conference Edition, November, 2019}

The sediments were smeared on a clean greasefree glass slide. After air drying and heat fixing, the smeared slide was stained by hot ZiehlNeelsen (ZN) staining technique for the detection of acid fast bacilli (AFB). The sediments were also cultured on Lowenstein Jensen (LJ) egg medium. The cultured medium was incubated at $37^{\circ} \mathrm{C}$ for up to six weeks.

Confirmation and Molecular Detection of Rifampicin Resistant M. tuberculosis (RRTB)

Molecular detection of $M$. tuberculosis and RRTB was performed using Xpert ${ }^{\circ}$ MTB/RIF according to the manufacture's instruction. The Xpert $\AA$ MTB/RIF purifies and concentrates $M$. tuberculosis bacilli from clinical samples. Genomic material isolated from the captured bacteria by sonication and subsequently amplifies the genomic DNA by polymerase chain reaction (PCR). Furthermore, the process identifies all the clinically relevant rifampicin resistance inducing mutations in the RNA polymerase beta $(r p o B)$ gene in the $M$. tuberculosis genome in a real time format using fluorescent probes called molecular beacons (Mulu et al., 2017).

\section{Determination of Immune Parameters}

Four (4) mls of venous blood was drawn aseptically from anterior cubital vein by means of vacutainer syringe from the selected participants and transferred into vacutainer tube containing potassium ethylene diamine tetra acetic acid ( $\mathrm{K}_{2}$ EDTA) as described by Cheesbrough (2006). All the samples collected were processed within four hours and analyzed on the same day. The patient's immune parameters (CD4, CD8, CD45 and CD3) were determined from the blood samples collected on flow cytometry (Becton-Dickson FACSCalibur, San Jose CA).

Two sets of Tri-Test reagent kits: one containing $\mathrm{CD} 3 / \mathrm{CD} 4 / \mathrm{CD} 45$ and the other containing CD3/CD8/CD45 were used. Phycoerythrin (PE) tagged against monoclonal antibody (MoA) CD4, Allophycocyanin (APC) tagged against MoA CD8, Peridinin-chlorophyll protein (Per CP) tagged against MoA CD45, and Fluorescein isothiocyanate (FITC) tagged against (MoA) $\mathrm{CD} 3$, were used for absolute count of $\mathrm{CD} 4+$, CD8+, CD45+ and CD3+ lymphocytes respectively.

Intradermal Tuberculin Skin Test (TST)

Intra dermal Tuberculin Skin Test (TST) was performed by intradermal injection on the forearm of 5 TU $(0.1 \mathrm{ml})$ of PPD and the indurations read as described by Centers for Disease Control and Prevention (CDC) (2013). This is followed by the LTBI group which recorded a CD4+, CD8+, CD3+and CD45+ cell.
Reactions are classified as positive if induration measures $\geq 10 \mathrm{~mm}$ indicates delayed-type hypersensitivity and also indicates past or present infection with M. tuberculosis. Whereas, induration $\leq 5 \mathrm{~mm}$ indicates lack of DTH to PPD and tuberculosis infection is highly unlikely.

\section{RESULTS}

The result of the study indicated that among the 205 studied subjects, 46 (22\%) were control subjects, $40(19.5 \%)$ were LTBI subjects, 64 $(31.7 \%)$ were DS-TB patients and $54(26.3 \%)$ were patients with RR-TB. The results further revealed that 131 of the studied subjects were males and 74 were females (Table 1). The Table also shows that among the 46 patients identified with drug susceptible TB (DS-TB) $70.8 \%$ were males while $29.2 \%$ were females. Similarly, among the 54 patients with rifampicin resistant TB (RR-TB), $66.7 \%$ were males and $33.3 \%$ were females respectively (Table 1 ).

Table 2 shows that majority of the studied subjects were in the age group 14-24 years $(41.0 \%), 25-34$ years $(35.6 \%)$ and $35-44$ years $(13.7 \%)$ respectively. The Table further shows that majority of the patients identified with DSTB were aged $14-24$ years (40\%) and $25-34$ years $(30.7 \%)$. Similarly, among the 54 patients with RR-TB, most of them were aged 14-24 years $(24.1 \%)$ and 25 -34years $(46.4 \%)$ (Table 2).

Table 3 revealed that $86.2 \%$ of the samples from the DS-TB were identified as AFB positive, while $88.9 \%$ of the RR-TB samples were AFB positive. Table 3 further shows that all the 65 samples of DS-TB and 54 samples of the RR-TB were identified as MTB by culture. Similarly, all the 65 DS-TB samples were detected as susceptible TB by Xpert ${ }^{\circledR}$ MTB/RIF, and all the 54 samples of the RR-TB were identified as rifampicin resistant by the Xpert ${ }^{\circledR}$ MTB/RIF.

Table 4 revealed that the mean T-cell (CD4+, CD8+, CD3+ and CD45+) counts of the studied subjects varied significantly among the different groups of the study $(p<0.05)$ and that compared to the control and the LTBI groups, the DS-TB and the RR-TB groups recorded the lowest counts. Least significance difference (LSD) indicated a significance difference in the CD4+, CD8+, CD3+ and CD45+ cells counts of the studied subjects in the order; Control>LTBI>DSTB $>$ RR-TB. Specifically, the Table indicated that the highest mean $\mathrm{CD} 4+, \mathrm{CD} 8+, \mathrm{CD} 3+$ and CD45+ cell count of $1081.6 \mathrm{cells} / \mu \mathrm{l}$, 529.0cells/ $\mu \mathrm{l}, 994.93 \mathrm{cells} / \mu \mathrm{l}$ and $1854.28 \mathrm{cells} / \mu \mathrm{l}$ were recorded from the control group. 
Special Conference Edition, November, 2019

The DS-TB group recorded a CD4+, CD8+, $\mathrm{CD} 3+$ and CD45+ cell count 684.23cells/ $\mathrm{\mu l}$, 411.11cells $/ \mu \mathrm{l}, \quad 882.40 \mathrm{cells} / \mu \mathrm{l} \quad$ and 1308.78cells/ $\mu$ l respectively (Table 4 ). For, the

RR-TB groups $\mathrm{CD} 4+, \mathrm{CD} 8+, \mathrm{CD} 3+$ and $\mathrm{CD} 45+$ cell counts were 497.26 cells/ $\mu \mathrm{l}, 352.0$ cells $/ \mu \mathrm{l}$, $700.78 \mathrm{cells} / \mu \mathrm{l}$ and $994.3 \mathrm{cell} / \mathrm{s} / \mathrm{l}$ respectively (Table 4).

Table 1: Sex distribution of the studied subjects

\begin{tabular}{llllll}
\hline Sex & \multicolumn{4}{c}{ Tuberculosis Status of the Studied Subjects } & Total \\
& Control & LTBI & DS-TB & RR-TB & \\
\hline Males & $25(54.3 \%)$ & $24(60 \%)$ & $46(70.8 \%)$ & $36(66.7 \%)$ & $131(63.9 \%)$ \\
Females & $21(45.7 \%)$ & $16(40 \%)$ & $19(29.2 \%)$ & $18(33.3 \%)$ & $74(36.1 \%)$ \\
& $\mathbf{4 6 ( 2 2 . 4 \% )}$ & $\mathbf{4 0}(\mathbf{1 9 . 5 \% )}$ & $\mathbf{6 5}(\mathbf{3 1 . 7 \% )}$ & $\mathbf{5 4}(\mathbf{2 6 . 3 \% )}$ & $\mathbf{2 0 5 ( 1 0 0 \% )}$ \\
\hline
\end{tabular}

Key: LTBI-Latent TB Infection; DS-Drug Susceptible; TB-Tuberculosis; RR-Rifampicin resistance

Table 2: Age distribution of the studied subjects

\begin{tabular}{llllll}
\hline Age & \multicolumn{3}{c}{ Tuberculosis Status of the Studied Subjects } & Total \\
& Control & LTBI & DS-TB & RR-TB & \\
\hline $14-24$ & $30(65.2 \%)$ & $15(37.5 \%)$ & $26(40 \%)$ & $13(24.1 \%)$ & $84(41.0 \%)$ \\
$25-34$ & $12(26.1 \%)$ & $16(40 \%)$ & $20(30.7 \%)$ & $25(46.3 \%)$ & $73(35.6 \%)$ \\
$35-44$ & $4(8.7 \%)$ & $5(12.5 \%)$ & $9(13.8 \%)$ & $10(18.5 \%)$ & $28(13.7 \%)$ \\
$45-54$ & 0 & $2(5 \%)$ & $7(10.8 \%)$ & $5(9.3 \%)$ & $14(6.8 \%)$ \\
$>55$ & 0 & $25(5 \%)$ & $3(4.6 \%)$ & $1(1.6 \%)$ & $6(2.9 \%)$ \\
Total & $\mathbf{4 6}$ & $\mathbf{4 0}$ & $\mathbf{6 5}$ & $\mathbf{5 4}$ & $\mathbf{2 0 5}$ \\
\hline
\end{tabular}

Key: LTBI-Latent TB Infection; DS-Drug Susceptible; TB-Tuberculosis; RR-Rifampicin resistance

Table 3: Acid Fast Bacilli (microscopy), culture and RR-TB (Xpert) Status of the Studied Subjects

\begin{tabular}{lllllll}
\hline \multicolumn{1}{c}{ GROUP } & \multirow{2}{*}{ AFB } & \multicolumn{2}{c}{ CULTURE } & \multicolumn{2}{c}{ Xpert } \\
& Positive & Negative & Positive & Negative & Positive & Negative \\
\hline $\begin{array}{l}\text { Control } \\
(\mathbf{n}=\mathbf{4 6})\end{array}$ & 0 & $46(100 \%)$ & 0 & $46(100 \%)$ & 0 & $46(100 \%)$ \\
LTBI $(n=40)$ & 0 & $40(100 \%)$ & 0 & $40(100 \%)$ & 0 & $40(100 \%)$ \\
DS-TB $(n=65)$ & $56(86.2 \%)$ & $9(13.8 \%)$ & $65(100 \%)$ & 0 & 0 & $65(100 \%)$ \\
RR-TB $(n=54)$ & $48(88.9 \%)$ & $6(11.1 \%)$ & $54(100 \%)$ & 0 & $54(100 \%)$ & 0 \\
Total $(\mathbf{n = 2 0 5 )}$ & $\mathbf{1 0 4}$ & $\mathbf{1 0 1}$ & $\mathbf{1 1 9}$ & $\mathbf{8 6}$ & $\mathbf{5 4}$ & $\mathbf{1 5 1}$ \\
& $\mathbf{( 5 0 . 7 \% )}$ & $\mathbf{( 4 9 . 3 \% )}$ & $\mathbf{( 5 8 \% )}$ & $\mathbf{( 4 1 . 2 \% )}$ & $\mathbf{( 5 6 . 6 \% )}$ & $\mathbf{( 4 3 . 4 \% )}$ \\
\hline
\end{tabular}

Key: AFB-Acid fast bacilli; LTBI-Latent TB Infection; DS-Drug Susceptible; TB-Tuberculosis; RR-

Rifampicin resistance

Table 4: Mean T-cell (CD4+, CD8,$+ C D 3+$ and $C D 45+)$ counts of the studied subjects

\begin{tabular}{lllll}
\hline $\begin{array}{l}\text { Tuberculosis Status } \\
\text { of the Studied Subjects }\end{array}$ & CD4+ & Mean T-cell counts $($ cells/ $\boldsymbol{\mu l})$ \\
\hline Control $(n=46)$ & 1081.36 & 529.0 & CD3+ & CD45+ \\
LTBI $(n=40)$ & 690.03 & 405.10 & 994.93 & 1854.28 \\
DS-TB $(n=65)$ & 684.23 & 411.11 & 883.53 & 1525.83 \\
RR-TB $(n=54)$ & 497.26 & 352.0 & 700.78 & 1308.78 \\
\hline
\end{tabular}

Key: AFB-Acid fast bacilli; LTBI-Latent TB Infection; DS-Drug Susceptible; TB-Tuberculosis; RRRifampicin resistance

Note: $*$ F-calculated $=29.249, \mathrm{~F}_{\mathrm{df}=3 ; 0.05}=2.604$, therefore $\mathrm{p}<0.05$

*Least significance difference (LSD) indicated a significance difference in the CD4+, CD3+ and CD45+ cells counts of the studied subjects in the order: Control>LTBI >DS-TB>RR-TB and the LSD indicated a significance difference in the CD8+ cells counts of the studied subjects in the order: Control $>$ LTBI $>$ RR-TB and Control $>$ DS-TB $>$ RR-TB. 


\section{DISCUSSION}

The findings of this study revealed that among both the DS-TB and the RR-TB patients more males were infected with TB than females and this coincides with the WHO (2018) reports that men are more affected with TB than women. In an earlier study, Neyrolles and Quintana-Murci (2009) reported a male/female ratio of $1.9 \pm 0.6$ among TB patients. Neyrolles and QuintanaMurci (2009) further pointed out this excess of male pulmonary TB cases is seen in all regions of the world, and in almost all countries, at least in non-HIV-infected patients. They further expounded that large prevalence surveys have suggested that the sex bias observed in pulmonary TB cases may result partly from genuine biological differences in male and female susceptibility to $M$. tuberculosis infection or the development of TB disease. According to Davila et al. (2008), several other, more specific biological sex-related factors that may render men even more susceptible to pulmonary TB than women include: sex steroid hormones, the genetic makeup of the sex chromosomes, and sex-specific metabolic features.

The study demonstrates that majority of the infected patients are aged between 14 years to 44years. This may not be unconnected with the fact that patients in this category are physically active and may be engaged in various sectors of life working experiences as such becoming more exposed to acquire and develop TB. The WHO (2018) reports that $90 \%$ of TB is seen among adults (aged $\geq 15$ years).

The study revealed that the traditional culture method still remains the gold standard diagnostic procedure for identification of TB as all the DS-TB and RR-TB samples were identified as MTB by culture, whereas $86.2 \%$ and $88.9 \%$ of the samples from the DS-TB and the RR-TB samples were AFB positive respectively. Similarly, the Xpert ${ }^{\circ}$ MTB/RIF performed excellently as all the DS-TB samples were detected as susceptible TB and all the RR-TB samples were identified as rifampicin resistant. The observation of this study is comparable to that of Roce et al. (2017) which shows that, the Xpert MTB/RIF revealed sensitivity and specificity of $89.6 \%$ and $97.2 \%$ for the detection of MTBC and $100 \%$ sensitivity and $98.3 \%$ specificity for the detection of Rifampicin Resistance. Other studies such as those by Rahman et al. (2016); Sharma et al. (2015) also agree with the observations of this study. In their contribution, Arend and van Sooligen (2017) opined that the implementation of Xpert MTB/RIF since 2010 has revolutionized molecular diagnosis of TB which combines early diagnosis of TB with direct detection of Rifampicin Resistance. They further stated that to overcome some of its limitations, the performance of Xpert MTB/RIF was reengineered to increase its diagnostic sensitivity and specificity in the detection of Rifampicin Resistance in form of MTP/RIF Ultra (Xpert Ultra).

Mycobacterium tuberculosis resides in lung phagocytes and can persist in its host for decades, and apart from macrophages which are the most abundant host cells at sites of infection which have been implicated in both disease control and progression (Huang et al. 2018), foremost in the anti-mycobacterial immune responses are also $\mathrm{T}$ lymphocytes especially $\mathrm{CD} 4+$ and $\mathrm{CD} 8+\mathrm{T}$ cells which produce various cytokines including IFN-y and display cytolytic activity against mycobacteria infected cells (Weiss and Schaible, 2015). The present study also demonstrated the presence of various $T$ cell subsets among the studied subjects with CD45+ T cells having higher counts compared to CD4+, CD8+ and CD3+ T cells.

The study further demonstrated that the mean T-cell (CD4+, CD8+, CD3+ and CD45+) counts varied significantly $(p<0.05)$ among the different groups of the study and that compared to the control (healthy subjects) and the LTBI groups the DS-TB and the RR-TB groups recorded the lowest counts. The observation that the mean $T$ cell counts of the control and LTBI subjects were found to be significantly higher than those of DS-TB and RR-TB subjects implies that there is profound immunological activity involving the $T$ cells denoting accumulation of higher bacterial load in the DS-TB and RR-TB subjects compared to the Control and LTBI subjects. These observations are consistent with earlier studies by Ordway et al. (2007) which revealed that during the acute and sub-acute stages of TB infection the immune response involve the recruitment of more $\mathrm{CD} 4+\mathrm{T}$ cells, including cells expressing the activation marker of CD45, but few CD8+ T cells. They however show that soon afterwards (after 30days) a dramatic loss in the CD4 response occurs. Furthermore, in support of the hypothesis of an augmented lymphocyte pooling in the site of infection, Venturini et al. (2019) revealed that there was a reduction of CD3+ and CD4+ absolute and percentage depletion in active TB (ATB) patients versus healthy control $(\mathrm{HC})$ subjects in their study which was found to be consistent with earlier studies. 


\section{Special Conference Edition, November, 2019}

This study further emphasized the role played by CD4+ $T$ cells in controlling the activity of other cells directed at Mycobacterial infection. For example, as the CD4+ $T$ cell count decreases among the various studied subjects the CD8+ T cell count also decreases. Studies by Yao et al. (2014) revealed that CD4+ T cells are needed to prevent rapid TB progression and to sustain multiple effector functions of CD8+ T and NKlike cells during $M$. tuberculosis infection of nonhuman primates. They demonstrated that CD4+ $\mathrm{T}$ cell depletion during $M$. tuberculosis infection leads to early extrapulmonary $M$. tuberculosis dissemination, a subsequent loss of systemic/pulmonary responses of multiple effector cells, and rapid progression to more severe TB in macaques.

Finally, the findings of this study implies that assessment of $\mathrm{T}$ cells sub sets particularly CD4+, $C D+8, C D 45$ and $C D 3 T$ cells may be used in the prognosis of DR-TB as evidenced by the least significance difference (LSD) which indicated a significance difference in the $\mathrm{CD} 4+, \mathrm{CD} 8+$, CD3+ and CD45+ cells counts of the studied

\section{REFERENCES}

Arend, S. M. and van Soolingen, D. (2017). Performance of Xpert MTB/RIF Ultra: a matter of dead or alive. Lancet, 18 (1): 8-10.

DOI:https://doi.org/10.1016/S14733099(17)30695-3

Centers for Disease Control and Prevention (CDC) (2013). Mantoux tuberculin skin test. https://www.cdc.gov.

Cheesbrough, M. (2006). District Laboratory Practice in Tropical Countries. $5^{\text {th }}$ ed. Cambridge: Cambridge University Press.

Davey, B. (1993). Immunology: a foundation text. England: John Wiley \& Sons, Ltd.

Davila, S., Hibberd, M. L., Dass, R. H., Wong, H. E. E., Sahiratmadja, E., Bonnard, C., Alisjahbana, B., Szeszko, J. S., Balabanova, Y., Drobniewski, F., van Crevel, R., van de Vosse, E., Nejentsev, S., Ottenhoff, T. H. M. Aand Seielstad, M. (2008). Genetic Association and Expression Studies Indicate a Role of Toll-Like Receptor 8 in Pulmonary Tuberculosis. PLOS Genetics 4 (10): e1000218

https://doi.org/10.1371/journal.pgen.10 00218

Deveci, F., Akbulut, H. H., Celik, I., Muz, M. H. and 'Ilhan, F. (2006). Lymphocyte Subpopulations in Pulmonary Tuberculosis Patients. Mediators of Inflammation, 2006 (89070): 1-6. DOI $10.1155 / \mathrm{MI} / 2006 / 89070$. subjects in the order; Control $>$ LTBI $>$ DS-TB $>$ RRTB.

\section{CONCLUSION}

The study revealed that males had the highest number of cases and patients aged 21-30 years and 31-40 years had the highest number of cases. The study also revealed that the T-cell counts of the studied subjects varied significantly $(p<0.05)$ among the different groups of the study and that compared to the control and the LTBI groups, the DS-TB and the RR-TB groups recorded the lowest counts. This implies that assessment of $T$ cells sub-sets may be used in the prognosis of DR-TB which will enhance early diagnosis \& treatment of TB as well as strengthen the TB control strategy.

\section{Acknowledgement}

The authors acknowledge the management of Infectious Diseases Hospital, Kano for the use of their facility.

\section{Conflict of interest}

None.

Doherty, T. M. and Andersen, P. (2005). Vaccines for tuberculosis: novel concepts and recent ogress. Clinical Microbiology Reviews, 18 (4): 687-702. doi: 10.1128/CMR.18.4.687-702.2005.

Dorhoi, A., Nouailles, G., Jo"rg1, S., Hagens, K., Heinemann, E., Pradl, L., OberbeckMu"ller, D., Duque-Correa1, M. A., Reece, S. T., Ruland, J., Brosch, R., Tschopp, J., Gross, O. and Kaufmann, S. H. E. (2012). Activation of the NLRP3 inflammasome by Mycobacterium tuberculosis is uncoupled from susceptibility to active tuberculosis. Eur. J. Immunol. 42: 374-384. http:// www.eji-journal.eu

Fayyazi, A., Eichmeyer, B., Soruri, A., Schewyer, S., Herms, J., Stefan, Jochen, S., Schwarz, P. and Heinz J. R. (2005). Apoptosis of macrophages and T cells in tuberculosis associated caseous necrosis. Journal of Pathology, 191 (4): 417-425. https://doi.org/10.1002/10969896(2000)9999:9999<::AIDPATH664>3.0.CO;2-R

Huang, L., Nazarova, E. V., Tan, S., Liu, Y. and Russell, D. G. (2018). Growth of Mycobacterium tuberculosis in vivo segregates with host macrophage metabolism and ontogeny. Journal of Experimental, 215 (4): 1135-1152. https://doi.org/10.1084/jem.20172020

Kaufmann, S. H. (2013). Tuberculosis vaccines: 
Semin Immunol. 25 (2): 172-81. Doi:10.1016/j.smim.2013.04.006.

Lawson, L., Habib, A. G., Okobi, M. I., Idiong, D., Olajide, I., Emenyonu, N., Onuoha, N., Cuevas, L. E. and Ogiri, S. O. (2010): Pilot study on multidrug resistant tuberculosis in Nigeria. Annals of African Medicine 9 (3):184-7

McNerney, R., Maeurer, M., Abubakar, I., Marais, B., McHugh, T. D., Ford, N., Weyer, K., Lawn, S., Grobusch, M. P., Memish, Z., Squire, S. B., Pantaleo, G., Chakaya, J., Casenghi, M., Migliori, G. B., Mwaba, P., Zijenah, L., Hoelscher, M., Cox, H., Swaminathan, S., Kim, P. S., Schito, M., Harari, A., Bates, M., Schwank, S., O'Grady, J., Pletschette, M., Ditui, L., Atun, R. and Zumla, A. (2012). Tuberculosis diagnostics and biomarkers: needs, challenges, recent advances, and opportunities. J Infect Dis, 205 (Suppl 2): S147-58. doi:10.1093/infdis/jir860.

Mulu, W., Abera, B., Yimer, M., Hailu, T., Ayele, $H$. and Abate, D. (2017). Rifampicin- resistance pattern of Mycobacterium tuberculosis and associated factors among presumptive tuberculosis patients referred to Debre Markos Referral Hospital, Ethiopia: a cross- sectional study. BMC Res Notes, 10:8. DOI 10.1186/s13104-016-2328-4

National Committee for Clinical Laboratory Standards (NCCLS). Susceptibility of Mycobacteria, Norcadia, and Actinomycetes $2^{\text {nd }}$ ed. Tentative Standards M24-T2 Wayne, P.A.7. 2011

National Tuberculosis and Leprosy Control Programme (NTBLCP) (2011): Manual on Technical SOPs for Tuberculosis Laboratories in Nigeria.

NIPOST (2008): Post offices with maps of LGAs. Retrieved from; http://www.nipost.gov.ng/postcode.aspx

Neyrolles, O. and Quintana-Murci, L. (2009). Sexual Inequality in Tuberculosis. PLOS Medicine, 6 (12):e1000199. DOI:10.1371/journal.pmed.1000199

Ordway, D., Palanisamy, G., Henao-Tamayo, M., Smith, E. E., Shanley, C., Orme, I. M. and Basaraba, J. B. (2007). The cellular immune response to Mycobacerium tuberculosis infection in the guinea pig. $J$ Immunol, 179: 2532-2541.

Perreau, M., Rozot, V., Welles, H. C., BellutiEnders, F., Vigano, S., Maillard, M., Dorta, G., Mazza-Stalder, J., Bart, P., Roger, T., Calandra, T., Nicod, L. and Harari, A. (2013). Lack of
Mycobacterium tuberculosis-specific interleukin-17A-producing CD4+ T cells in active disease. Eur $\mathrm{J}$ Immunol, 43: 939-948 DOI: 10.1002/eji.201243090.

Rahman, A., Sahrin, M., Afrin, S., Earley, K., Ahmed, S., Rahman, S. M. M., et al. (2016) Comparison of Xpert MTB/RIF Assay and GenoType MTBDRplus DNA Probes for Detection of Mutations Associated with Rifampicin Resistance in Mycobacterium tuberculosis. PLOS ONE 11 (4): e0152694. doi:10.1371/journal.pone.0152694.

Roce, J. P., Seifert, M., Moser, K. S. and Rodwell, T. C. (2017). Perfomance of Xpert MTB/RIF assay for the diagnosis of pulmonary tuberculosis and rifampicin resistance in low-incidence, high resource setting. PLOS ONE 12 (10): e0186139. doi: 10.137/ journal.pone.0186139.

Schoenborn, J. R. and Wilson, C. B. (2007). Regulation of interferon-gamma during innate and adaptive immune responses. Adv Immunol. 96:41-101. DOI: 10.1016/S0065-2776(07)96002-2

Sharma, S. K., Kohli, M., Yadav, R. N., Chaubey, J., Bhasin, D., Sreenivas, V., Sharma, R. and Singh, B. K. (2015) Evaluating the Diagnostic Accuracy of Xpert MTB/RIF Assay in Pulmonary Tuberculosis. PLOS ONE $10 \quad$ (10): $\quad$ e0141011. doi:10.1371/journal.pone.0141011

Venturini, E., Lodi, L., Francolino, I., Ricci, S., Chiappini, E., de Martino, M. and Galli, L. (2019). CD3, CD4, CD8, CD19 and CD16/CD56 positive cells in tuberculosis infection and disease: Peculiar features in children. International Journal of Immunopathology and Pharmacology, 33: 1-13. DOI: $10.1177 / 2058738419840241$

Weiss, G. and Schaible, U. E. (2015). Macrophage defense mechanisms against intracellular bacteria. Immunol Rev, $264 \quad$ (1): http://doi.org/10.1111/imr.12266

Wiley, J. M., Sherwood, L. M. and Woolverton, C. J. (2008). Prescott, Harley and Klein's Microbiology. New York: McGraw-Hill Companies.

World Health Organization (2017). Global Tuberculosis Report. WHO/HTM/TB/2017.23

World Health Organization (2018). Global Tuberculosis WHO/CDS/TB/2018.20 
Yao, S., Huang, D., Chen, C. Y., Halliday, L., Wang, R. C. and Chen, Z. W. (2014). CD4+ $T$ Cells Contain Early Extrapulmonary Tuberculosis (TB) Dissemination and Rapid TB Progression and Sustain Multieffector Functions of CD8+ $\mathrm{T}$ and CD32 Lymphocytes: Mechanisms of CD4+ T Cell Immunity. $J$ Immunol, 192: 2120-2132. http://www.jimmunol.org/content/192/5 $\angle 2120$.

Zumla, A., Atun, R., Maeurer, M., Mwaba, P., Ma, Z., O'Grady, J., Bates, M., Dheda, K., Hoelscher, M. and Grange, J. (2011). Scientific dogmas, paradoxes and mysteries of latent Mycobacterium tuberculosis infection. Tropical Medicine and International Health, 16 (1): 79-83. doi:10.1111/j.1365-3156.2010.02665.x 